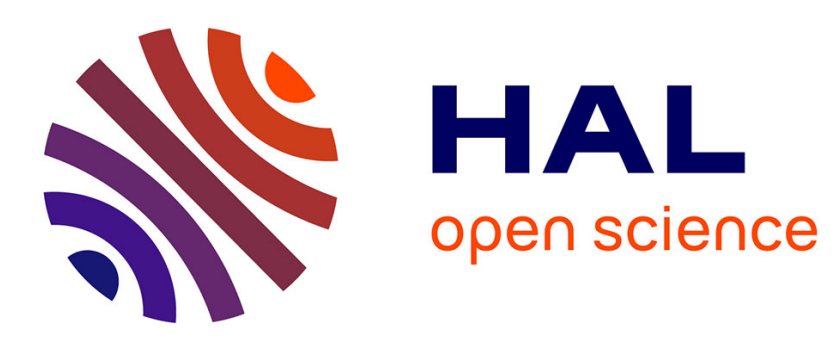

\title{
Asymétries de la fonction explicative des représentations intergroupes hommes/femmes
}

Élise Vinet, Pascal Moliner

\section{To cite this version:}

Élise Vinet, Pascal Moliner. Asymétries de la fonction explicative des représentations intergroupes hommes/femmes. Les cahiers Internationaux de Psychologie Sociale, 2006, 69, pp.47 - 57. 10.3917/cips.069.0047 . halshs-01561804

\section{HAL Id: halshs-01561804 https://shs.hal.science/halshs-01561804}

Submitted on 13 Jul 2017

HAL is a multi-disciplinary open access archive for the deposit and dissemination of scientific research documents, whether they are published or not. The documents may come from teaching and research institutions in France or abroad, or from public or private research centers.
L'archive ouverte pluridisciplinaire HAL, est destinée au dépôt et à la diffusion de documents scientifiques de niveau recherche, publiés ou non, émanant des établissements d'enseignement et de recherche français ou étrangers, des laboratoires publics ou privés. 


\title{
ASYMÉTRIES DE LA FONCTION EXPLICATIVE DES REPRÉSENTATIONS INTERGROUPES HOMMES/FEMMES
}

Élise Vinet, Pascal Moliner

\author{
Presses universitaires de Liège | «Les Cahiers Internationaux de Psychologie \\ Sociale »
}

2006/1 Numéro 69 | pages 47 à 57

ISSN 0777-0707

Article disponible en ligne à l'adresse :

http://www.cairn.info/revue-les-cahiers-internationaux-de-psychologiesociale-2006-1-page-47.htm

\section{Pour citer cet article :}

Élise Vinet, Pascal Moliner « Asymétries de la fonction explicative des représentations intergroupes hommes/femmes », Les Cahiers Internationaux de Psychologie Sociale 2006/1 (Numéro 69), p. 47-57.

DOI 10.3917/cips.069.0047

Distribution électronique Cairn.info pour Presses universitaires de Liège.

(c) Presses universitaires de Liège. Tous droits réservés pour tous pays.

La reproduction ou représentation de cet article, notamment par photocopie, n'est autorisée que dans les limites des conditions générales d'utilisation du site ou, le cas échéant, des conditions générales de la licence souscrite par votre établissement. Toute autre reproduction ou représentation, en tout ou partie, sous quelque forme et de quelque manière que ce soit, est interdite sauf accord préalable et écrit de l'éditeur, en dehors des cas prévus par la législation en vigueur en France. Il est précisé que son stockage dans une base de données est également interdit. 


\title{
Asymétries de la fonction explicative des représentations intergroupes hommes/femmes
}

\author{
Élise VINET et Pascal MOLINER \\ Laboratoire de psychologie sociale, Université Paul Valéry - Montpellier III, France
}

\begin{abstract}
Résumé : Dans cette recherche, menée sur 324 sujets en 3 phases, on s'intéresse aux représentations de genre endogroupe et exogroupe d'étudiants de premier cycle en faculté de lettres. Les résultats suggèrent une plus grande homogénéité perçue de la cible féminine, i.e., de l'endogroupe des femmes et de l'exogroupe des hommes. Ils suggèrent en outre que ces représentations de la cible féminine ont une fonction explicative nettement plus marquée que les représentations de la cible masculine, i.e., de l'endogroupe des hommes et de l'exogroupe des femmes. Ces résultats sont discutés au regard de la notion d'attribution sociale et de la fonction explicative des stéréotypes.
\end{abstract}

Mots-clés : représentations intergroupes, homogénéité perçue, asymétrie positionnelle, attribution.

Le savoir naif n'est jamais indépendant des contextes sociaux dans lesquels il est produit et mis en œuvre. C'est un savoir dont la géométrie peut tout autant varier en fonction de ceux qui l'utilisent qu'en fonction des objets qu'il concerne. Ce phénomène d'«ancrage psychosocial » (Doise, 1992), a reçu plusieurs illustrations empiriques (Tafani et Bellon, 2001) qui suggèrent que, selon un principe d'homologie structurale (Bourdieu, 1977 ; Lorenzi- Cioldi, 1988), les représentations sont partiellement déterminées par les positions de domination ou de subordination des individus qui les élaborent. Ce principe pose une correspondance entre structure sociale et structures cognitives des individus, les secondes résultant de la position qu'occupent les individus dans la première : «le statut agit sur la manière dont les individus se représentent le groupe, sur la manière dont ils se conçoivent et conçoivent autrui, et sur les rapports que leur groupe entretient avec d'autres groupes » (Lorenzi-Cioldi et Doise, 1994, p. 91). C'est originellement Bourdieu $(1977,1998)$ qui pose, dans sa théorie des champs sociaux, l'espace social comme un système organisé de positions sociales déterminées par trois dimensions : le capital économique, qui renvoie aux ressources matérielles des individus ; le capital culturel, qui réfère à un niveau de compétence sanctionné par un diplôme, et le genre, posé comme une dimension fondamentale du positionnement social.
Les individus s'inscrivent donc dans un ensemble «d'asymétries positionnelles reflétant les valeurs de pouvoir qui structurent notre société, les enjeux qu'elles actualisent et les luttes qu'elles occasionnent » (Tafani et Bellon, 2001, p. 163).

\section{Représentations intergroupes, homogénéités et hétérogénéité perçues}

On sait que dans leurs interactions, les groupes élaborent des savoirs sur eux-mêmes et sur les autres. Pour qualifier ces savoirs, des auteurs tels que Doise (1973), ou Deschamps (1973), ont introduit la notion de représentation intergroupe (RIG). Pour Deschamps (1973, p. 715), une RIG «se forme au travers des jugements portés par des groupes sur d'autres, jugements eux- mêmes déterminés par la nature des rapports entre ces groupes ". Dans cette perspective, on pourrait avancer qu'en raison de leur ancrage psychosocial, les RIG contribuent à la pérennisation des situations de domination sociale : cf. notamment l'idéologie dominante de Bourdieu $(1977,1998)$ ainsi que la notion de system-justification de Jost et Banaji (1994). Cependant, une récente étude de Tafani, Audin et Apostolidis (2002) nuance ce principe de reproduction systémique de l'ordre social en montrant que les représentations sociales - au sens Moscovicien d' " univers d'opinions" portant sur tout objet social, animé ou non -, si elles contribuent au maintien par les groupes dominants de leur pouvoir social, permettent également aux groupes dominés de remettre en cause cette domination subie. Par ailleurs, de nombreux travaux (Linville, Salovey et Fischer, 1986; LorenziCioldi, 1988; Park, Ryan et Fudd, 1992) attestent d'un effet d'hétérogénéité perçue des groupes dominants, dits collections (Lorenzi-Cioldi et Doise, 1990 ; Lorenzi-Cioldi, 2002) au sein desquels les individus sont considérés comme distincts les uns des autres, uniques et de ce fait non interchangeables. Ces mêmes travaux attestent d'un effet concomitant d'homogénéité perçue des

Pour toute correspondance relative à cet article, s'adresser à Elise Vinet, Laboratoire de Psychologie Sociale, Université Paul Valéry - Montpellier III, Route de Mende, 34199 Montpellier cEDEx 5 ou par courriel à < elise.vinet@univ-montp3.fr>. 
groupes dominés, dits agrégats dont les membres sont considérés comme similaires les uns aux autres, et par conséquent largement interchangeables (voir aussi Scharnitsky, 1998, pour une revue complète de la question). Aussi, dans les recherches sur les représentations intergroupes hommes/femmes (cf. notamment l'étude de Lorenzi-Cioldi, Eagly et Steward, 1995), il est fait état d'une «tendance des hommes comme des femmes à homogénéiser le groupe féminin, i.e., un effet d'homogénéisation de l'exogroupe pour les hommes, et un effet d'homogénéisation de l'endogroupe

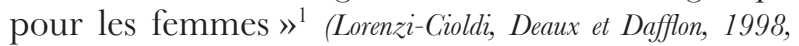
p. 256). Or cette homogénéisation porte directement atteinte à l'estime de soi, puisque comme le rappelle Lorenzi-Cioldi (2002, p. 93) "l'idéologie occidentale est individualiste : le groupe y est vécu comme un dilueur de la personnalité »; elle va donc consacrer «le propotype d'une personne autonome, autodéterminée et douée d'unicité » (ibid, p.174, voir aussi Smith et Zarate, 1992). À ce propos, une étude de Sachdev et Bourhis (1991) montre que les groupes de haut statut jouissent du pouvoir social - au sens de «degré de contrôle dont bénéficie un groupe sur sa propre destinée et sur celle de l'exogroupe » (fones, 1972, p.183) - et d'une identité plus positive que les groupes de bas statut, déindividués, donc culturellement dévalorisés et ainsi exposés à la discrimination évaluative (Wilder, 1986).

Cette asymétrie dans les perceptions réciproques des groupes trouve de multiples explications. L'hétérogénéisation - i.e. la connaissance différenciée et approfondie des dominants par les dominés relèverait, pour Bourdieu (1990, p.24) d'une « lucidité des exclus ». Pour Park, Ryan et Judd (1992, p. 566), elle relèverait d'un mécanisme "plus utile » à ces derniers. Pour Brown (1990, p. 230) elle serait « nécessaire à leur survie » (voir aussi l'hypothèse de l'oppression, Henley, 1973 ;1977, LaFrance et Henley, 1994), puisque le destin des dominés est lié, de par leur statut, à leur adaptation aux dominants (Fiske, 1993, Keltner et Robinson, 1997). Enfin, les membres des groupes dominés seraient plus familiers (Pryor et Ostrum, 1987, Wilder, 1986) de ceux des groupes dominants que de leur propre endogroupe, en raison de la position de ces derniers dans l'espace social et de l'attention dont ils font ainsi l'objet de la part de tous (cf. l'hypothèse du contact, Linville et al., 1986, Linville, Fisher et Salovey, 1989).
Quant à l'homogénéisation dont les groupes dominés font preuve à leur propre endroit, elle tiendrait à plusieurs faits. Prisant le modèle d'identité des dominants, les groupes dominés intérioriseraient les bonnes raisons qui font d'eux ce qu'ils sont - des dominés - (Pinçon et Pinçon-Charlot, 2000), et adopteraient ainsi le point de vue véhiculé par les groupes dominants, dont le jugement fait office de référent (Lorenzi-Cioldi et Doise, 1994). Brewer (1993) et Simon (1992) considèrent quant à eux que cette homogénéisation peut être envisagée comme une tentative de restauration d'une identité menacée. Pour Mullen (1991), elle relèverait d'une cohésion favorisée par la faiblesse des stimulations sociales dont ces groupes font l'objet.

Concernant maintenant l'homogénéisation des groupes dominés par les groupes dominants, ces derniers maitrisant les interactions sociales avec les premiers (Messick et Mackie, 1989), elle pourrait être appréhendée comme une négligence, par les dominants, des caractéristiques de ceux dont ils contrôlent la destinée (Fiske, 1993), négligence liée à une absence de motivation à les connaître de façon détaillée (Lorenzi-Cioldi, 1998). N'oublions pas que d'une façon plus triviale, cette homogénéisation permet aussi aux dominants de maintenir leur prestige et d'assurer leur contrôle sur les dominés.

Enfin, la personnalisation (l'hétérogénéité) dont les groupes dominants font preuve à leur propre endroit constituerait pour le groupe une protection sociale stratégique en le rendant quasi-inattaquable, puisque constitué justement d'individus distincts, apparemment sans rapports les uns avec les autres (LorenziCioldi, 2002). D'autre part, elle assurerait, par un pied de nez, la légitimité de la domination du groupe - eu égard à la valorisation culturelle occidentale de l'unicité et de l'autodétermination - et donc sa pérennité... Au détriment des groupes défavorisés et en retour essentialisés. Néanmoins, comme le rappellent Deschamps et Beauvois (1994, p. 122) « en dépit de cette adhésion à des images susceptibles de les stigmatiser, les groupes minoritaires ou défavorisés peuvent adopter diverses stratégies pour protéger l'estime qu'ils ont d'eux-mêmes " (voir à ce propos Crocker et Major, 1989; Azzi et Klein, 1998 ; Lorenzi-Cioldi, 2002)².

1. "(...) a tendency for both men and women to homogenize the female group, i.e., an outgroup homogeneity effect for men and an ingroup homogeneity effect for women".

2. La classification classique pose 2 grands types de stratégies, les individuelles et les collectives. Dans les premières, on trouve diverses stratégies dont le changement de groupe d'appartenance lorsque cela est possible, ce qui n'est pas le cas des groupes d'appartenance sexués, ou encore l'effet P.I.P (Codol, 1975), qui consiste, grossièrement, à se considérer comme meilleur que les autres au sein même du groupe d'appartenance, donc à 
Au regard de ces diverses considérations, il semble donc que l'homogénéisation des groupes dominés n'entretiennent pas de lien causal avec un quelconque essentialisme, mais relève bien au contraire de la représentation que les individus se font du positionnement dominant ou dominé de leur propre groupe dans un espace social donné (cf.notamment Tafani et Deschamps, 2004, eu égard au statut social des individus dont l'effet prime sur leur statut sexué).

\section{Homologie structurale homogénéité perçue et fonction explicative des représentations intergroupes}

D’après Doise (1973), les représentations intergroupes (RIG) remplissent principalement deux fonctions : une fonction justificatrice qui permet de légitimer le comportement des membres d'un groupe à l'égard d'un autre. Et une fonction anticipatrice d'orientation des comportements intergroupes. Mais les RIG sont aussi des représentations sociales. En ce sens, on peut s'attendre à ce qu'elles assument donc les fonctions habituellement dévolues à ces dernières (Abric, 1994). On pense bien sûr ici à une fonction identitaire, mais surtout aux fonctions de compréhension et d'explication de l'environnement social. En effet, pour assumer leur fonction de «savoir» (Abric, 1994, p. 15), les RIG doivent fournir aux individus qui les utilisent des éléments explicatifs auxquels il est possible d'attribuer les causes des comportements, des prises de position ou des situations qui caractérisent l'endo-groupe ou l'exo-groupe (Deschamps, 1973). Ces attributions (Heider, 1958), permettent de rendre le monde contrôlable et prédictible, tout en maintenant et préservant un système de croyance existant.

Mais dans le cas des RIG, ces attributions ne sont jamais dissociées des insertions sociales. Ainsi que le notent Deschamps et Beauvois (1994, p. 125) «autrui semble perçu comme étant à la source de son comportement ou encore comme étant déterminé par la situation en fonction de son appartenance à certaines catégories qui ont une valeur spécifique aux yeux des sujets et des observateurs eux- mêmes socialement insérés ». La notion d'" attribution sociale ", proposée par Deschamps (1973; voir aussi Deschamps et Clémence, 1987), souligne ainsi l'interdépendance qui unit attribution causale, représentations intergroupes et relations intergroupes, en suggérant que l'individu pourrait chercher à expliquer le comportement d'un acteur non pas à partir des caractéristiques individuelles de cet acteur, mais à partir des caractéristiques associées au groupe social auquel cet acteur appartient. Dans cette perspective, on peut supposer que les RIG qui seront alors mobilisées vont jouer un rôle essentiel dans le processus d'attribution, en raison notamment des éléments explicatifs qu'elles peuvent suggérer aux individus. On trouve un bel exemple de ce phénomène dans la recherche de Deaux et Emswiller (1974), qui porte sur l'explication du succès dans une tâche. Lorsque cette tâche est typiquement masculine, ce qui est attribué à la compétence (attribution interne) pour un homme (de la part des hommes et des femmes) est attribué à la chance (attribution externe) pour une femme (toujours par consensus des deux sexes). Par contre, lorsque la tâche est typiquement féminine, les sujets continuent d'expliquer le succès d'un homme en termes de compétences. Partant, si les attributions intergroupes sont liées aux relations entre les groupes, on voit alors qu' « elles dépendent (...) des positions relatives qu'occupent ces groupes les uns par rapport aux autres. C'est-à-dire que le statut relatif des groupes en présence fait que, dans bien des cas, les attributions ne sont pas symétriques » (Deschamps, 1996, p. 258). On trouve d'autres exemples de l'effet du statut des groupes sur les processus d'attribution dans les travaux princeps de Geis (1993), Conway, Pizzamiglio et Mount (1996), puis dans ceux récents de Echebarria-Echabe et Fernandez (2002), travaux mettant en évidence le lien entre statut des groupes cibles et attribution de traits communautaires (communal traits, i.e. l'empathie, l'expression des sentiments... considérés comme plutôt féminins) ou instrumentaux (intrumental traits, i.e. l'indépendance, l'autorité... considérés comme plutôt masculins). Ces derniers travaux mettent de plus l'accent sur la prééminence du statut social sur le statut sexué (cf. aussi Tafani et Deschamps, 2004), en montrant que les sujets font preuve de plus d'attributions décisionnelles (instrumentales) concernant une personne, homme ou femme, occupant un poste de haut statut, qu'il s'agisse d'un

\footnotetext{
valider l'appartenance groupale - garante de la reconnaissance voir de la protection sociale- tout en s'en démarquant de par un surplus de qualité - garant d'une estime de soi préservée d'autant plus que le groupe d'appartenance est stigmatisé... Concernant les stratégies collectives, on y trouve notamment des stratégies de créativité sociale - changement de dimension de comparaison endo/exogroupe à l'avantage cette fois de l'endogroupe dominé- ou encore des stratégies de compétition sociale - dimension de comparaison conservée mais cette fois c'est la position des groupes en présence qui peut basculer, par des stratégies de discrimination notamment, en faveur de l'endogroupe dominé (voir notamment Brewer, 1979, études sur le PGM).
} 
poste à connotation féminine ou masculine (EchebarriaEchabe et Fernandez-Guede, 2002) ${ }^{3}$. Aussi, si l'on admet que les RIG contiennent des éléments explicatifs, on peut supposer que ces attributions générales, valables pour tous les membres d'un groupe, seront d'autant plus nombreuses et faciles à produire que les membres de ce groupe seront perçus comme très semblables. Dans cette lignée, Deschamps et Beauvois (1994, pp. 125-126) s'attendent «à ce que l'information catégorielle imprègne d'autant plus les attributions que la cible fait partie d'un groupe perçu comme très homogène, ce qui est plus souvent le cas $(. .$.$) d'un groupe dominé que d'un groupe$ dominant ». Or, on sait que les asymétries de statut contribuent à la perception de l'homogénéité des groupes dominés et de l'hétérogénéité des groupes dominants. Par conséquent, si l'on s'intéresse à des groupes dont on sait qu'ils occupent des positions distinctes dans l'espace social, comme c'est le cas des hommes et des femmes, mais dans un contexte de neutralité statutaire sociale - un amphithéâtre où tous les sujets ont le même statut d'étudiant afin que le statut sexué représente la seule asymétrie statutaire prégnante de cette population, on peut s'attendre à observer des asymétries dans la finalité explicative de leurs représentations réciproques. En d'autres termes, on peut faire l'hypothèse que l'effet d'homogénéité perçue s'accompagne d'une plus grande saillance des éléments explicatifs des représentations intergroupes. En théorie, dans le groupe dominant, la représentation de l'exo-groupe dominé, perçu comme homogène, devrait être plus «explicative» que la représentation de l'endogroupe, perçu comme hétérogène. À l'inverse, dans le groupe dominé, c'est la représentation de l'endo-groupe, perçu comme homogène, qui devrait être plus «explicative » que celle de l'exogroupe dominant, perçu comme hétérogène. La recherche qui suit a tenté de mettre cette hypothèse à l'épreuve.

\section{Méthodologie \\ Population et procédure}

Cette recherche a été menée auprès d'étudiants de premier cycle, inscrits en faculté de lettre. Elle s'est déroulée en trois étapes. La première étape a consisté à recueillir les contenus des représentations endogroupe et exo-groupe des hommes et des femmes « en général ». Pour cela, les sujets (109 femmes et
23 hommes) devaient répondre aux deux questions suivantes : "Quelles sont, selon vous les trois caractéristiques les plus fréquemment partagées par les hommes (vs les femmes) en général ? ». Nous avons donc sollicité une production stéréotypée des sujets, de par le contexte de comparaison sociale induit et contrôlé - tous les sujets répondant d'abord pour l'endogroupe -, et de par le contenu même de la question, puisqu'on demandait aux sujets de produire des caractéristiques «les plus fréquemment partagées ».

Selon cette procédure, nous avons recueilli un total de 792 mots. Selon le sexe des sujets et celui de la cible, nous avons alors constitué quatre corpus. Les deux premiers concernaient les réponses des sujets femmes à propos de l'endogroupe (131 mots différents) et de l'exogroupe (135 mots différents). Les deux suivants concernaient les réponses de sujets hommes à propos de l'endogroupe (34 mots différents) et de l'exogroupe (35 mots différents). Le traitement de ces quatre corpus s'est effectué séparément afin de tenir compte de la surreprésentation des sujets féminins dans notre population. Dans un premier temps, les mots constitutifs de chaque corpus ont été réduits à leur forme canonique et agrégés selon leur proximité sémantique. Dans un second temps, après analyse de contenu (D'Unrung, 1974, Muchielli, 1996, Bardin, 2001, Moliner, Rateau et Cohen-Scali, 2002), nous avons regroupé les mots en catégories thématiques. Enfin, dans chaque condition, nous avons sélectionné les items dont la récurrence était supérieure à 10\% du corpus par condition.

Ainsi, pour la cible « les hommes en général », nous avons retenu 13 items, dont 4 apparaissent à la fois dans les productions des sujets masculins et féminins (sexualité, sport, fierté et amitié), 4 sont spécifiques aux sujets masculins et 5 spécifiques aux sujets féminins. Pour la cible « les femmes en général », nous avons retenus 12 items, dont 4 sont données à la fois par les sujets masculins et féminins (coquetterie, communication, douceur et séduction, 6 sont spécifiques aux sujets masculins et 2 aux sujets féminins. On notera que l'item « sexualité » est le seul à être commun aux deux cibles. De sorte qu'au final, nous avons abouti à une liste de 24 items différents.

Dans une seconde étape, destinée à explorer les représentations intergroupes, les 24 items furent proposés à 120 sujets répartis en quatre sous groupes,

3. "High status activities (masculine or feminine) provoked stronger attribution of intrumentality than low status job" (Echebarria-Echabe et Fernandez, 2002, p. 110). 
Tableau 1 : Contenus des représentations des hommes vs femmes "en général". Fréquences d'apparition des items communs aux sujets masculins $(\mathrm{H})$ et féminins $(\mathrm{F})$ et des items spécifiques aux sujets masculins ou féminins

\begin{tabular}{|c|c|c|c|c|c|c|c|}
\hline & \multicolumn{3}{|c|}{ Items communs à tous les sujets } & \multirow{2}{*}{\multicolumn{2}{|c|}{$\begin{array}{l}\text { Items spécifiques } \\
\text { aux sujets masculins }\end{array}$}} & \multirow{2}{*}{\multicolumn{2}{|c|}{$\begin{array}{l}\text { Items spécifiques aux } \\
\text { sujets féminins }\end{array}$}} \\
\hline & $\mathrm{H}$ & & $\mathrm{F}$ & & & & \\
\hline \multirow{5}{*}{$\begin{array}{l}\text { LES HOMMES } \\
\text { EN GÉNÉRAL }\end{array}$} & $26 \%$ & Sexualité & $43 \%$ & Force & $24 \%$ & Fête & $30 \%$ \\
\hline & $25 \%$ & Sport & $26 \%$ & Frime & $11 \%$ & Violence & $17 \%$ \\
\hline & $16 \%$ & Fierté & $13 \%$ & Machisme & $10 \%$ & Compétition & $13 \%$ \\
\hline & $12 \%$ & Amitié & $26 \%$ & Ambition & $10 \%$ & Domination & $13 \%$ \\
\hline & & & & & & Individualisme & $13 \%$ \\
\hline \multirow{6}{*}{$\begin{array}{l}\text { LES FEMMES } \\
\text { EN GÉNÉRAL }\end{array}$} & $36 \%$ & Coquetterie & $30 \%$ & Enfants & $25 \%$ & Sexualité & $22 \%$ \\
\hline & $17 \%$ & Communication & $13 \%$ & Sensibilité & $25 \%$ & Dépendance & $17 \%$ \\
\hline & $13 \%$ & Douceur & $17 \%$ & Jalousie & $19 \%$ & & \\
\hline & $13 \%$ & Séduction & $13 \%$ & Altruisme & $17 \%$ & & \\
\hline & & & & Hypocrisie & $10 \%$ & & \\
\hline & & & & Affectivité & $10 \%$ & & \\
\hline
\end{tabular}

selon le plan Sujets <Sexe2 * Cible2>. Les sujets, hommes ou femmes, devaient se prononcer à propos du groupe des hommes ou des femmes en choisissant 12 items qui, selon eux « caractérisaient le mieux » les membres du groupe cible. Nous avons opté pour cette procédure de choix forcé car elle est couramment utilisée dans les études de représentation (Abric, 2003), particulièrement dans les études comparatives. En effet, cette méthode permet de proposer le même questionnement à tous les sujets. Par ailleurs, puisque la phase exploratoire a permis d'identifier 12 items pour chaque cible, il nous a paru judicieux de proposer aux sujets l'ensemble des 24 items puis de leur demander d'en choisir 12. Précisons que pour neutraliser un éventuel effet d'ordre, la liste d'items était aléatoirement présentée dans deux ordres différents.

Dans une troisième étape, destinée à repérer les items explicatifs des différentes représentations intergroupes explorées, 72 sujets étaient répartis en 4 sous groupes, selon le plan Sujets $<$ Sexe2 ${ }^{*}$ Cible2>. Les sujets, hommes ou femmes, devaient se prononcer à propos du groupe cible des hommes ou des femmes en choisissant 12 items qui, selon eux « expliquaient le mieux l'attitude » des membres du groupe cible. Là encore, la liste d'items était présentée selon deux ordres différents.

\section{Hypothèses}

$H_{1}$ : Effets des asymétries statutaires sur les processus d'homogénéité perçue

Sur la base des résultats robustes obtenus dans de nombreux travaux précédents (cf. Lorenzi-Cioldi, 2002, pour une revue très complète de la question), ainsi qu'au regard plus général des phénomènes d'ancrage psychosocial et d'homologie structurale, on s'attend d'une part à ce que la représentation endogroupe des sujets féminins soit plus homogène que leur représentation de l'exogroupe masculin, et d'autre part à ce que la représentation endogroupe des sujets masculins soit moins homogène que leur représentation de l'exogroupe féminin.

\section{$H_{2}$ : Effets de l'homogénéité perçue sur la fonction explicative des $R I G$}

Compte tenu de l'homogénéité attendue de la représentation endogroupe des femmes et exogroupe des hommes, et selon les travaux invoqués supra relatifs à l'articulation entre homogénéisation et attribution sociale (Deschamps et Beauvois, 1994, Lorenzi-Cioldi et al., 1998, Echebarria-Echabe et Fernandez-Guede, 2002), on s'attend à ce que chez les femmes, les éléments explicatifs soient plus saillants dans la représentation endogroupe que dans la représentation de l'exogroupe masculin (dominant). Inversement chez les hommes : on s'attend à ce que les éléments explicatifs soient moins saillants dans la représentation endogroupe que dans la représentation de l'exogroupe féminin (dominé).

\section{Résultats \\ Les représentations intergroupes hommes/femmes, validation du matériel}

Dans la présente recherche, nous ne commenterons pas les contenus des différentes représentations explorées. Conformément aux hypothèses énoncées plus haut, nous nous contenterons de nous centrer sur la question de l'homogénéité de ces représen- 
Tableau 2 : Qu'est-ce qui caractérise le mieux les femmes vs les hommes en général ? Fréquences des choix d'items selon le sexe des sujets

\begin{tabular}{lcccc}
\hline \multicolumn{1}{c}{ SuJETs } & \multicolumn{2}{c}{$\mathrm{F}$} & \multicolumn{2}{c}{$\mathrm{H}$} \\
\hline CIBLE & $\mathrm{F}$ & $\mathrm{H}$ & $\mathrm{H}$ & $\mathrm{F}$ \\
\hline douceur & 0,87 & 0,07 & 0,20 & 0,93 \\
enfant & 0,87 & 0,33 & 0,03 & 0,93 \\
affectivité & 0,97 & 0,33 & 0,30 & 0,90 \\
sensibilité & 0,97 & 0,37 & 0,27 & 0,87 \\
communication & 0,93 & 0,40 & 0,37 & 0,90 \\
ambition pro & 0,80 & 0,33 & 0,50 & 0,60 \\
altruisme & 0,80 & 0,47 & 0,50 & 0,80 \\
jalousie & 0,23 & 0,13 & 0,20 & 0,23 \\
séduction & 0,67 & 0,63 & 0,53 & 0,77 \\
hypocrisie & 1 & 0,90 & 0,70 & 0,93 \\
dépendance & 0,20 & 0,20 & 0,23 & 0,27 \\
sexualité & 0,43 & 0,47 & 0,43 & 0,37 \\
aitié & 0,73 & 0,97 & 0,80 & 0,80 \\
force & 0,73 & 0,70 & 0,73 & 0,50 \\
compétition & 0,43 & 0,60 & 0,80 & 0,40 \\
individualisme & 0,37 & 0,80 & 0,70 & 0,37 \\
fête & 0,13 & 0,27 & 0,40 & 0,17 \\
fierté & 0,10 & 0,70 & 0,67 & 0,50 \\
domination & 0,33 & 0,93 & 0,87 & 0,23 \\
sport & 0,17 & 0,67 & 0,67 & 0,17 \\
frime & 0,20 & 0,57 & 0,70 & 0,10 \\
violence & 0 & 0,47 & 0,50 & 0,23 \\
machisme & 0,07 & 0,30 & 0,50 & 0 \\
\hline & 0 & 0,40 & 0,40 & 0,03 \\
\hline
\end{tabular}

tations et sur leur finalité explicative. Le tableau 2 présente les fréquences de choix d'items selon le sexe des sujets et celui de la cible, en réponse au questionnaire de caractérisation (étape 2).

Dans un premier temps, nous avons voulu nous assurer de la fiabilité de notre questionnaire. Cependant compte tenu du fait que ce questionnaire est constitué d'items «masculins » et «féminins », il était impossible de lui appliquer un indice de fiabilité tel que l'alpha de Cronbach. En effet, par construction, notre questionnaire est sensé mesurer deux choses différentes. Il était donc nécessaire de diviser le questionnaire en deux listes, correspondant chacune aux caractéristiques attribuées aux cibles féminines et masculines. Afin d'échapper à l'inévitable subjectivité de notre analyse de contenu initiale, nous avons donc procédé à une analyse factorielle des correspondances sur les données du tableau 2.
Figure 1 : Représentation graphique des axes 1 et 2 de l'AFC réalisée sur les données du tableau

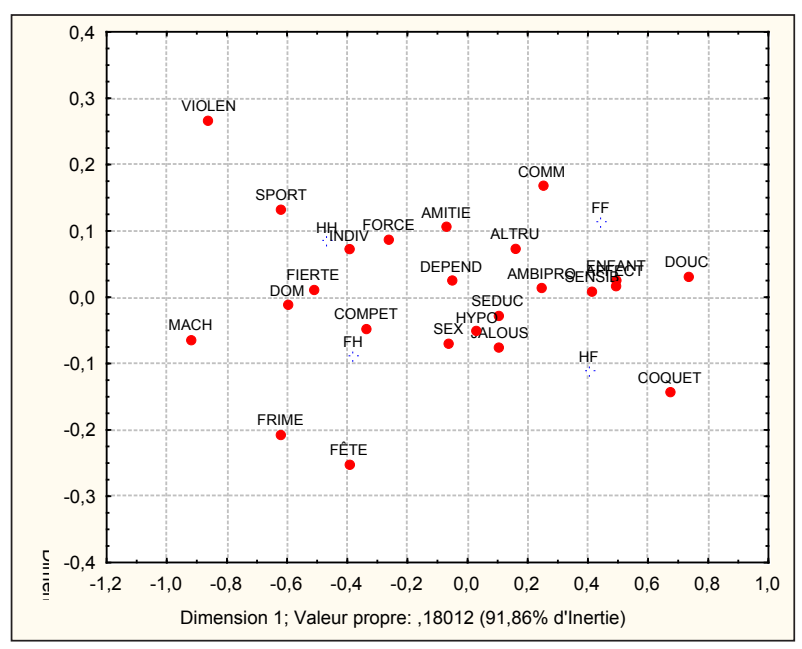

Comme on peut facilement le constater sur la figure 1, le premier facteur de l'analyse, qui rend compte de $91.86 \%$ de l'inertie totale, oppose nettement les cibles masculines (à gauche de la figure, dénommées $\mathrm{HH}-$ « hommes » (sexe des sujets) sur « hommes » (sexe de la cible) - et FH - «femmes » sur « hommes »- aux cibles féminines (à droite, dénommées $\mathrm{FF}$ et $\mathrm{HF}$ ). Il s'agit donc d'un facteur opposant des items «masculins » (machisme, violence, sportif, domination) à des items "féminins » (douceur, coquetterie, enfants, sensibilité). Nous avons donc considéré que les 12 items ayant les coordonnées les plus négatives sur ce facteur étaient des items masculins, tandis que les 12 items ayant les coordonnées les plus positives étaient des items féminins. L'alpha de Cronbach, calculé sur la liste d'items masculins s'élève à .65 et il s'élève à .62 pour la liste d'items féminins, ce qui témoigne d'une bonne fiabilité du questionnaire.

\section{Homogénéité perçue des groupes cibles}

Afin d'apprécier l'homogénéité perçue des groupes cibles, nous avons considéré, pour chacune des 4 conditions, les 12 items ayant obtenu les plus fortes fréquences de choix. En théorie, selon la procédure que nous avons adoptée, si un groupe cible est perçu comme totalement homogène par l'ensemble des sujets d'une condition donnée, on devrait observer 12 items ayant obtenu $100 \%$ des choix des sujets. À l'inverse, si ce groupe cible est perçu comme totalement hétérogène, on devrait observer une fréquence de $50 \%$ des choix des sujets pour les 24 items du questionnaire. Ainsi, dans une condition donnée, les 12 items ayant obtenu les plus fortes fré- 
Tableau 3 : scores moyens d'homogénéité perçue selon le sexe des sujet et celui du groupe cible

\begin{tabular}{cccc}
\hline \multirow{2}{*}{ SUJET } & & \multicolumn{2}{c}{ CiBLE } \\
& & Endo & Exo \\
\hline \multirow{2}{*}{ F } & 9,76 & 8,40 \\
& $\mathrm{H}$ & 8,16 & 9,43 \\
\hline
\end{tabular}

quences de choix constituent un repère à partir duquel il est possible d'estimer l'homogénéité perçue du groupe cible. Nous avons donc calculé pour chaque sujets et dans chaque condition, un score d'homogénéité perçue. Ce score correspond donc au nombre d'items choisis par un sujet qui appartiennent à la liste des 12 items les plus choisis par le groupe. Il peut varier de 0 à 12 . Lorsqu'il est égal à 12, cela signifie que le sujet caractérise les membres du groupe cible de la même manière que la majorité des autres sujets placés dans la même condition. Lorsqu'il est égal à 0 , cela signifie que le sujet caractérise les membres du groupe cible totalement différemment de la majorité des autres sujets placés dans la même condition. Enfin, nous avons calculé un score moyen d'homogénéité pour chacune des 4 conditions : lorsque la moyenne des scores individuels, pour une condition donnée, se rapproche de 12, cela signifie que la plupart des sujets de cette condition caractérisent les membres du groupe cible avec les mêmes items. Donc qu'ils les perçoivent comme très ressemblants. Sur le tableau 3 , on peut voir les scores moyens d'homogénéité perçue selon le sexe des sujets et celui de la cible.

L'analyse de variance réalisée sur ces scores moyens $\left(\right.$ S30<Sexe2 ${ }^{*}$ Cible2>) ne fait apparaître aucun effet simple. En revanche on constate un fort effet d'interaction $(F(1-116)=29.55, p<.0001)$. L'analyse des contrastes confirme que les femmes se perçoivent comme un groupe plus homogène qu'elles ne perçoivent le groupe cible des hommes (9.76 vs 8.40 $F(1-116)=13.61, p<.0004)$. Au contraire, les hommes se perçoivent comme un groupe moins homogène qu'ils ne perçoivent le groupe cible des femmes (8.16 vs $9.43 ; F(1-116)=15.92, p<.0002)$. Ce résultat confirme donc notre première hypothèse.

Pour aller plus loin, nous avons voulu savoir si le phénomène d'homogénéité perçue ne reposait pas sur des items spécifiques aux groupes et aux cibles en présence. Pour cela, nous avons pris en considération les résultats de l'étude exploratoire (cf. tableau 1), afin de calculer des scores partiels d'homogénéité. Cependant, nous n'avons pu mettre en évidence
Tableau 4 : Qu'est-ce qui explique le mieux l'attitude des femmes vs les hommes en général ? Fréquences des choix d'items selon le sexe des sujet

\begin{tabular}{|c|c|c|c|c|}
\hline \multirow{2}{*}{$\begin{array}{c}\text { SUJETS } \\
\text { CIBLE }\end{array}$} & \multicolumn{2}{|c|}{$\mathrm{F}$} & \multicolumn{2}{|c|}{$\mathrm{H}$} \\
\hline & $\mathrm{F}$ & $\mathrm{H}$ & $\mathrm{H}$ & $\mathrm{F}$ \\
\hline force & .35 & .88 & .85 & 0 \\
\hline sport & .06 & .82 & .95 & 0 \\
\hline domination & .12 & .82 & .60 & .16 \\
\hline frime & .06 & .70 & .65 & .33 \\
\hline fête & .17 & .59 & .75 & .39 \\
\hline machisme & 0 & .59 & .70 & .16 \\
\hline douceur & 1 & .48 & .10 & .83 \\
\hline sensibilité & .94 & .35 & .20 & .89 \\
\hline affectivité & .88 & 0 & .25 & .89 \\
\hline coquetterie & .82 & 0 & 0 & .94 \\
\hline enfants & .82 & .12 & .25 & .72 \\
\hline fierté & .47 & .94 & .90 & .61 \\
\hline violence & 0 & .29 & .35 & 0 \\
\hline individualisme & 0 & .53 & .30 & .16 \\
\hline hypocrisie & .47 & $; .35$ & .15 & .72 \\
\hline compétition & .59 & .82 & .80 & .16 \\
\hline communication & .82 & 0 & .35 & .33 \\
\hline amitié & .65 & .47 & .75 & .78 \\
\hline jalousie & .76 & .65 & .50 & 1 \\
\hline séduction & .94 & .94 & .65 & 1 \\
\hline altruisime & .29 & .17 & .15 & .28 \\
\hline ambition & .65 & .59 & .75 & .44 \\
\hline dépendance & .35 & .17 & .25 & .44 \\
\hline sexualité & .76 & 1 & .80 & .72 \\
\hline
\end{tabular}

aucun résultat significatif. On peut supposer que cette absence de résultat est liée à la faiblesse des différences initiales entre les items.

\section{Fonction explicative des représentations intergroupes hommes/femmes}

Le tableau 4 présente les résultats obtenus à partir du questionnaire "explicatif » (étape 3). Dans un premier temps, nous avons considéré tous les items ayant été choisis par au moins $50 \%$ des hommes et $50 \%$ des femmes à propos d'une même cible. Pour chacun de ces items, nous avons ensuite vérifié que la fréquence des choix à propos de cette cible était statistiquement supérieure à la fréquence des choix pour la cible opposée $\left(\chi^{2}, p<.05\right)$, autant chez les sujets hommes que chez les sujets femmes. Par convention, nous avons appelé "Attributions Majoritaires » (AM) les items répondant à ces critères. Ainsi, les AM Masculines sont : Force, Sport, 
Tableau 5 : scores explicatifs moyens selon le sexe des sujets et celui du groupe cible

\begin{tabular}{lccc}
\hline & & \multicolumn{2}{c}{ Cible } \\
& & Endo & Exo \\
\hline \multirow{2}{*}{ SUJET } & $\mathrm{F}$ & .92 & .56 \\
& $\mathrm{H}$ & .62 & .90 \\
\hline
\end{tabular}

Domination, Frime, Fête et Machisme. En moyenne, chacun de ces items est choisi par $75 \%$ des hommes et $73 \%$ des femmes pour expliquer la cible masculine. Chacun d'entre eux est en moyenne choisi par $17 \%$ des hommes et $12 \%$ des femmes pour expliquer la cible féminine. Les AM Féminines sont : Douceur, Sensibilité, Affectivité, Coquetterie et Enfant. En moyenne, chacun de ces items est choisi par $85 \%$ des hommes et $89 \%$ des femmes pour expliquer la cible féminine. Chacun d'entre eux est en moyenne choisi par $16 \%$ des hommes et $9 \%$ des femmes pour expliquer la cible masculine.

Enfin, nous avons calculé un score «explicatif» pour chaque sujet, dans chaque condition. Ce score résulte du nombre d'AM choisies par un sujet lorsqu'il décrit une cible à l'aide du questionnaire de caractérisation (étape 2). Selon le sexe de la cible, il repose sur les AM Masculines ou Féminines. Compte tenu du nombre différent d'AM dans les deux blocs, le score explicatif est divisé par le nombre respectif d'items qui le composent (6 AM Masculines et 5 Féminines). Il varie donc de 0 à 1 . Globalement, ce score reflète la saillance des items explicatifs relatifs à une cible dans la représentation de cette cible. Le tableau 5 présente les scores explicatifs moyens observés dans les différentes conditions de l'étude.

L'analyse de variance (S30<sexe2*cible2>), réalisée sur les scores explicatifs moyens observés dans les 4 conditions fait apparaître un net effet d'interaction $(F(1-116)=95.62, p<.00001)$. Pour caractériser l'endogroupe, les femmes choisissent nettement plus d'AM que ne le font les hommes (.92 vs .62 $F(1-116)=41.69, p<.00001)$. Mais pour caractériser l'exogroupe, ce sont les hommes qui choisissent le plus d'AM (.90 vs .56, $F(1-116)=54.35, p<.00001)$. En d'autres termes, il apparaît ici une asymétrie dans la fonction explicative des différentes représentations intergroupes sous étude, ce qui penche en faveur de notre deuxième hypothèse. Mais il reste à établir le lien entre l'asymétrie des attributions et l'asymétrie de l'homogénéité perçue des groupes.

\section{Homogénéité perçue et fonction explicative}

Si l'on considère que les valeurs du tableau 4 reflètent le "potentiel explicatif » de chaque item, alors il devient possible d'estimer la saillance globale de la fonction explicative des RIG hommes-femmes toutes conditions confondues. Pour cela, lorsqu'un sujet décrit une cible à l'aide du questionnaire de caractérisation (étape 2), on peut faire la somme du «potentiel explicatif » des 12 items qu'il a choisis. En théorie, cette somme peut varier de 0 à 12 . Elle sera égale à 12 lorsque les items choisis pour caractériser une cible (étape 2) ont été considérés comme explicatifs par tous les sujets lors de l'étape 3. Nous avons donc calculé un score «explicatif global» pour chacun des 120 sujets ayant répondu au questionnaire de caractérisation de l'étape 2. Rappelons que nous avions précédemment calculé pour chaque sujet, selon son sexe et celui de la cible, un score moyen d'homogénéité perçue. Ainsi, sur l'ensemble des 120 sujets de l'étape 2, nous avons finalement calculé la corrélation entre chaque «score explicatif global » et chaque score d'homogénéité perçue. On constate un lien très net $(r=.82, p<.001)$ entre homogénéité perçue et fonction explicative des représentations intergroupes sous étude. Ainsi, plus un groupe est perçu comme homogène, plus la fonction explicative de la représentation de ce groupe est saillante.

\section{Conclusion}

Au terme de cette recherche, il apparaît que nos hypothèses se trouvent confirmées.

En effet, nous avons tout d'abord retrouvé l'effet classique d'homogénéité perçue des groupes dominés et d'hétérogénéité perçue des groupes dominants. Nos résultats suggèrent en outre que l'homogénéité d'une représentation intergroupe peut faciliter le caractère explicatif de cette dernière. De ce point de vue, il nous semble que nos résultats apportent une contribution originale à la problématique de l'attribution sociale soulevée par Deschamps et Clémence (1987). En effet, ainsi que le note Deschamps (1996, p. 254) : «...les recherches qui abordent explicitement cette problématique de l'attribution entre groupes sont relativement peu nombreuses ». Or, dans la présente recherche, si nous avons pu voir que les représentations intergroupes hommes/femmes remplissaient bien une fonction explicative, nos résultats suggèrent que cette fonction n'est pas de même intensité pour les groupes en présence. Selon nous, les différences observées s'expliquent d'abord par le caractère plus ou moins 
homogène de ces représentations et finalement par le rapport asymétrique dans lequel se placent les groupes étudiés. Par ailleurs, ces résultats suggèrent que le phénomène d'attribution sociale pourrait reposer sur la présence d'éléments explicatifs au sein même des représentations intergroupes. Mobiliser ces représentations reviendrait donc à mobiliser les explications qu'elles contiennent.

Dans une autre perspective, il nous semble que les résultats de cette étude viennent illustrer la conception « explicative » des stéréotypes (Hoffman et Hurst, 1990). Selon ces auteurs, les stéréotypes ne se caractériseraient pas simplement par leur contenu évaluatif.Ilsseraientaussides explicationspermettant aux individus de comprendre ou de justifier pourquoi les membres d'un groupe se ressemblent et forment une catégorie. Dans cette même lignée, ils constitueraient d'après certains travaux fost et Banaji, 1994; Sidanius et Pratto, 1999) un support idéal de légitimation du statut social supérieur des groupes dominants, posant que ce ne sont ni les privilèges ni donc l'appartenance aux groupes dominants qui engendrent les compétences de ces derniers, mais l'inverse, i.e. leurs privilèges découleraient de leurs caractéristiques internes et personnelles. Cette fausse conscience (fost et Banaji, 1994) serait ensuite propagée aux dominés et adoptée par ces derniers, pour les raisons évoquées supra. En d'autres termes, en pouvant notamment jouer un rôle de justificateur du statut quo, les éléments stéréotypés d'une catégorie rempliraient une fonction explicative. Nous retrouvons ce phénomène dans nos résultats. En effet, si l'on examine les corrélations entre les fréquences de choix observés pour le questionnaire de caractérisation (étape 2) et celles observées pour le questionnaire explicatif (étape 3), on constate des coefficients très élevés. Ainsi, lorsque les sujets féminins se prononcent sur l'endogroupe, la corrélation entre les deux questionnaires ${ }^{4}$ et de .95 $(p<.01)$. Lorsque ces mêmes sujets se prononcent sur l'exogroupe, elle est de .67 $(p<.01)$. Dans le même sens, lorsque les sujets masculins se prononcent sur l'endogroupe, la corrélation entre les deux questionnaires est de $.90(p<.01)$ et elle est de .81 $(p<.01)$ lorsqu'ils se prononcent sur l'exogroupe. La réponse positive à une question posée a posteriori à un groupe d'étudiants ${ }^{5}$, ainsi que les corrélations présentées ci-dessus, certes significatives mais pas parfaites $(r=1)$ - notamment pour la condition exogroupe -, nous ont fait écarter l'hypothèse selon laquelle les deux questionnaires mesureraient la même chose. Au contraire, ils montrent, d'après nous, que plus un item est choisi pour caractériser les membres d'un groupe donné - particulièrement dans la condition endogroupe -, plus cet item est jugé explicatif de l'attitude des membres de ce groupe, et vice versa. Ainsi, bien que nous ayons pu mettre en évidence des différences dans la fonction explicative des représentations étudiées, il semble que pour tous les sujets interrogés, la description des membres de l'endogroupe ou de l'exogroupe contienne toujours une part d'explication.

Par ailleurs, au regard de notre population d'étude (jeunes étudiants et étudiantes), on peut supposer que cette homogénéisation consensuelle du groupe des femmes relève surtout du fait que les 2 groupes sexués ont été soumis à nos questionnaires certes dans l'anonymat et en passation individuelle avec consignes écrites, mais en présence mutuelle... ce qui a pu générer un phénomène comparable à celui de menace du stéréotype (Aronson et Steele, 1995 ; cf. aussi Desert, $2003)^{6}$. Les groupes n'ayant pas de tâche à résoudre, il se peut que cette menace ai simplement agit comme catalyseur d'une comparaison intergroupe explicite. Or, dans un cadre d'égalité statutaire manifeste - dans l'accès au savoir -, cette comparaison renvoyait alors directement aux asymétries statutaires sexuées, et donc à la domination masculine ${ }^{7}$. Cependant, on peut s'interroger sur l'apparente absence d'impact du surnombre des femmes dans les amphithéâtres de lettre... majorité numérique qui n'a apparemment pas contrebalancé leur infériorité statutaire sexuée. En effet, des questionnaires (étape 2 et 3) en nombre limité - conditions expérimentales $2 \times 2$ - et donc insuffisant, ont été répartis aléatoirement dans les amphithéâtres, jusqu'au plafond de sujets dans chaque condition, les autres sujets étant simplement observateurs. On pourrait donc

\footnotetext{
4. Ici, en respectant l'ordre des items, on calcule les corrélations entre les valeurs du tableau 1 et celles du tableau 4, colonne par colonne.

5. « Faites-vous la différence entre caractériser une attitude et expliquer une attitude ? » $\mathcal{N}=25$, dont 18 filles et 7 garçons. 23 sujets ont répondu oui et ont su argumenter, 2 filles ont répondu oui sans pouvoir argumenter.

6. Le fait de se trouver dans des situations où ils désirent ne pas confirmer des stéréotypes négatifs peut influencer négativement le fonctionnement cognitif des individus.

7. À condition que l'on considère que les étudiantes sont conscientes de l'infériorité statutaire des femmes et de l'homogénéisation représentationnelle qui en découle, d'un « toutes les mêmes » en somme, induisant, par un processus cognitif complexe, une confirmation du stéréotype menaçant... et une réaffirmation de ce stéréotype de la part des étudiants.
} 
supposer, dans le cas présent, que l'effet du genre ait été plus prégnant que l'effet du nombre pendant la passation, dans un contexte d'égalité statutaire apparente puisque tous les sujets étaient étudiants de même niveau dans le même cours. Cette hypothèse reste à être mise à l'épreuve dans des travaux ultérieurs.

\section{RÉFÉRENCES}

- Abric J.-C. (1994): Les représentations sociales : aspects théoriques. In J.-C. Abric (Dir.), Pratiques Sociales et Représentations. Paris, Presses Universitaires de France, pp. 11-36.

- Azzi A. E. et KLein O. (1998): Psychologie sociale et relations intergroupes. Paris, Dunod.

- Bardin L. (2001): L'analyse de contenu. Paris, Presses Universitaires de France.

- Bourdieu P. (1977): La production des croyances : contribution à une économie des liens symboliques. Actes de la Recherche en Sciences Sociales. 13, pp. 3-43.

- Bourdieu P. (1990): La domination masculine. Actes de la recherche en Sciences Sociales, 84, pp. 3-31.

- Bourdieu P. (1998): La domination masculine. Paris, Seuil.

- Brauer M., Guinote A. et Judd A. M. (2002): Effects of power on perceived and objective group variability: Evidence that more powerful groups are more variable. Fournal of Personality and Social Psychology, 82, pp. 708-721.

- Brewer M. B. (1979): Ingroup bias and the Minimal Group paradigm: A cognitive-motivational Analysis. Psychological Bulletin, 86, pp. 307-324.

- Brewer M. B. (1993): Social identity, distinctiveness, and in-group homogeneity. Social Cognition, 11, pp. 150-164.

- Brown L. S. (1990): What female therapists have in common. In D. W. Cantor (Dir.), Women as therapists. New York, Springer, pp. 227-242.

- Codol J.-P. (1975): PIP effect (Primus Inter Pares, first among aquals) and norm conflict. L'Année Psychologique, 75, pp. 127-145.

- Conway M., Pizzamiglio M. T. et Mount L. (1996): Status, communality, and Implications for stereotypes of gender and other groups. Fournal of Personality and Social Psychology, 71, pp. 25-38.

- Crocker J. et Major B. (1989): Social stigma and self-esteem: The selfprotective properties of stigma. Psychological Reviere, 96, pp. 608-630.

- Deaux K. et Emswiller T. (1974): Explanations of successful performance on sex-linked tasks: What is skill for the male is luck for a female. Fournal of Personality and Social Psychology, 29, pp. 80-85.

- Deschamps J.-G. (1973): L'attribution, la catégorisation sociale et les représentations intergroupes. Bulletin de psychologie, 13-14, pp. 710-721.

- Deschamps J.-G. (1996): Au delà des théories «classiques» de l'attribution. In J.-C. Deschamps et J.-L. Beauvois (Dirs.), La psychologie sociale, 2. Des attitudes aux attributions. Grenoble, Presses Universitaires de Grenoble, pp. 237-260.

- Deschamps J.-C. et Beauvois J.-L. (1994): Attributions intergroupes. In R.Y. Bourhis et J.-P. Leyens (Dirs.), Stéréotypes, discrimination et relations intergroupes. Sprimont, Mardaga, pp. 97-126.

- Deschamps J.-G et Clé́mence A. (1987): L'explication quotidienne. Perspectives psychososiologiques. Cousset, Delval.

- Désert M. (2003): La menace du stéréotype. In J.-C. Croizet et J.-P. Leyens (Dirs.), Stigmatisation et exclusion sociale. Paris, Armand Colin.

- Dorse W. (1973): Rencontres et représentations intergroupes. Archives de Psychologie, 61, pp. 303-320.

- Doise W. (1992): L'ancrage dans les études sur les représentations sociales. Bulletin de psychologie, 405, pp. 189-195.
- D’Unnung M. C. (1974), Analyse de contenu et acte de parole. Paris, Éditions Universitaires.

- Echebarria-Echabe A. et Fernandez-Guede E. (2002): Social determinants of attribution of instrumental and communal traits: Status, occupation, sex, and performance. Revue Internationale de Psychologie Sociale, Vol. 15, No2, pp. 91-116.

- Fiske S. T. (1993): Controlling other people. The impact of power on stereotyping. American Psychologist, 48, pp. 621-628.

- GeIs F. L. (1993): Self-fulfiling prophecies: A social psychological view of gender. In A. E. Beall et R. J. Sternberg (Dirs.), The psychology of gender. New York, The Guilford Press.

- Heider F. (1958): The psychology of interpersonal relations. New York, Wiley.

- Henley N. M. (1973): Status and sex: Some touching observations. Bulletin of the Psychonomic Society, 2, pp. 91-93.

- Henley N. M. (1977): Body politics : power, sex, and nonverbal communication. Englewood Cliffs, Prentice Hall.

- Hoffman C. et Hurst N. (1990): Gender stereotypes: Perception or rationalization? Fournal of Personality and Social Psychology, 58, pp. 197208.

- Jones J. M. (1972): Prejudice and Racism. Philadelphia, Addison Wesley.

- Jost J. T. et Banaji M. R. (1994): The role of stereotyping in systemjustification and the production of false consciousness. British fournal of Social Psychology, 33, pp. 1-27.

- Keltner D. et Robinson R. J. (1997): Defending the status quo: Power and bias in social conflict. Personality and Social Psychology Bulletin. 23, pp. 1066-1077.

- LaFrance M. et Henley N. M. (1994): On oppressing hypotheses ; or differences in nonverbal sensitivity revisited. In H. L. Radtke et H. J. Stam (Dirs.), Power/Gender: Social relations in theory and practice. Londres, Sage, pp. 287-311.

- Linville P. W., Fischer G. W. et Salovey P. (1989): Perceived distributions of the characteristics of in-group and out-group members: Empirical evidence and a computer simulation. Fournal of Personality and Social Psychology, 57, pp. 165-188.

- Linville P. W., Salovey P. et Fischer G, W. (1986): Stereotyping and perceived distributions of social characteristics: An application to in-group-out-group perception. In J. F. Dovidio et S. L. Gaertner (Dirs.), Prejudice, discrimination, and racism. San Diego, Academic Press, pp. 165-208.

- Lorenzi-Cioldi F. (1988): Individus dominants et groupes dominés. Grenoble, Presses Universitaires de Grenoble.

- Lorenzi-Cioldi F. (1998): Group status and perceptions of homogeneity. In W. Stroebe et M. Hewstone (Dirs.), European Review of Social Psychology, 9, pp. 31-75.

- Lorenzi-Cioldi F. (2002): Les représentations des groupes dominants et dominés, collections et agrégats. Grenoble, Presses Universitaires de Grenoble.

- Lorenzi-Gioldi F., Deaux K. et Dafflon A.-C. (1998): Group homogeneity as a function of relative social status. Sreiss fournal of Psychology, Vol. 57, N4, pp. 255-273. 
- Lorenzi-Cioldi F. et Doise W. (1990): Levels of analysis and social identity. In D. Abrams et M. Hogg (Dirs.), Social Identity Theory. New York, Harverster, pp. 71-88.

- Lorenzi-Cioldi F. et Doise W. (1994): Identité sociale et identité personnelle. In R. Y. Bouhris and J.-P. Leyens (Dirs.), Stéréotypes, discrimination et relations intergroupes. Sprimont, Mardaga, pp. 69-96.

- Lorenzi- Gioldi F., Eagly A. C. et Stewart T. L. (1995): Homogeneity of Gender Groups in Memory. Fournal of Experimental Social Psychology, 31, pp. 193-217.

- Moliner P., Rateau P. et Cohen-Scali V. (2002): Les représentations sociales, pratique des études de terrain. Rennes, Presses Universitaires de Rennes.

- Messick D. M. et Mackie D. M. (1989): Intergroup relations. Annual Review of Psychology. 40, pp. 45-81.

- Muchielli A. (1996): Dictionnaire des méthodes qualitatives en sciences humaines et sociales. Paris, Armand Colin.

- Mulden B. (1991): Group composition, salience, and cognitive representations: The phenomenology of being in a group. Fournal of Experimental Social Psychology, 27, pp. 297-323.

- Park B., Ryan C. S. et Judd C. M. (1992): Role of meaningful subgroups in explaining differences in perceived variability for ingroups and out-groups. Fournal of Personnality and Social psychology, 63, pp. 553-567.

- Pinçon M. et Pinçon-Charlot M. (2000): Sociologie de la bourgeoisie. Paris, La Découverte.

- Pryor J. B. et Ostrum T. M. (1987): Social cognition theory of group processes. In B. Mullen et G. R. Goethals (Dirs.), Theories of group behaviour. New York, Springer, pp.147-183.

- Sachdev I. et Bourhis R. Y. (1991): Power and status differentials in minority and majority group relations. European Fournal of Social Psychology, 21, pp. 1-24.
- Scharnitsky P. (1998): La variabilité perçue des groupes sociaux: Une revue de la question. Les Cahiers Internationnaux de Psychologie Sociale, 40, pp. 47-61.

- Sidanius J. et Pratto F. (1999): Social dominance: An intergroup theory of social hierarchy and oppression. Cambridge, Cambridge University Press.

- Simon B. (1992): The perception of ingroup and outgroup homogeneity Reintroducing the intergroup context. European Review of Social Psychology, Vol. 3, pp.1-30.

- Smith E. R. et Zàrate M. A. (1992): Exemplar-based models of social judgment. Psychological Review. 99, pp. 3-21.

- Steele C. M. et Aronson J. (1995): Stereotype threat and the intellectual test performance of African. Americans. Fournal of Personality and Social Psychology, Vol. 69, N5, pp. 797-811.

- Tafani É. et Bellon S. (2001): Principe d'homologie structurale et dynamique représentationnelle. In P. Moliner (Dir.), La dynamique des représentations sociales. Grenoble, Presses Universitaires de Grenoble, pp. 163-193.

- Tafani É., Audin S. et Apostolidis T. (2002): Asymétries positionnelles, identité sociale et dynamique représentationnelle: une étude expérimentale sur la représentation sociale des droits de l'Homme. Les Cahiers Internationaux de Psychologie Sociale. 54, 4.

- Tafani É. et Deschamps J.-G. (2004): Identité sociale et dynamique représentationnelle : positions sociales, menace identitaire et processus de différenciation. Nerw Review of Social Psychology, 3, pp. 38-46.

- WiLDER D. A. (1986): Social categorization: Implications for creation and reduction of intergroup conflict. In L. Berkowitz (Dir.), Advances in experimental social psychology, 19, pp. 291-355. San Diego, Academic Press. 